\title{
Myometrial contractility influences oxytocin receptor (OXTR) expression in term trophoblast cells obtained from the maternal surface of the human placenta
}

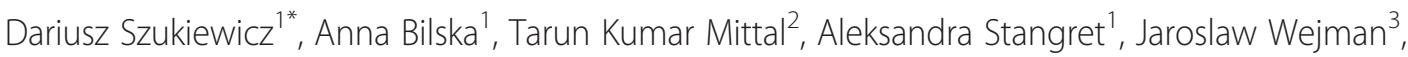
Grzegorz Szewczyk', Michal Pyzlak ${ }^{1,3}$ and Jacek Zamlynski ${ }^{4}$

\begin{abstract}
Background: Oxytocin (OXT) acts through its specific receptor (OXTR) and increased density of OXTR and/or augmented sensitivity to OXT were postulated as prerequisites of normal onset of labor. Expression of OXTR in the placental term trophoblast cells has not yet been analyzed in the context of contractile activity of the uterus. Here we examine comparatively OXT contents in the placental tissue adjacent to the uterine wall and expressions of OXTR in this tissue and corresponding isolated placental trophoblast cells.

Methods: Twenty eight placentae after normal labors at term (group I, $N=14$ ) and after cesarean sections performed without uterine contractile activity (group $\|, N=14$ ) have been collected. Tissue excised from the maternal surface of examined placenta was used for OXT concentration measurement, cytotrophoblast cell cultures preparation and immunohistochemistry of OXTR. Concentration of OXT was estimated in the tissue homogenates by an enzyme immunoassay with colorimetric detection. Cytotrophoblast cells were isolated using Kliman's method based on trypsin, DNase, and a 5-70 \% Percoll gradient centrifugation. The cultures were incubated for 5 days in normoxia. Both placental specimens and terminated cytotrophoblast cultures were fixed and embedded in paraffin before being immunostained for OXTR. Using light microscopy with computed morphometry for quantitative analysis, OXTR expressions were estimated in calibrated areas of the paraffin sections.

Results: There were not significant differences between the groups in respect to the mean OXT concentration. However, in both groups the median value of OXT concentration was significantly $(p<0.05)$ higher in the tissue obtained from the peripheral regions of the maternal surface of the placenta, compared to the samples from the central region of this surface. In placental tissue the mean expression of OXTR in group I was significantly $(p<0.05)$ increased by approximately 3.2-fold and 3.45-fold (the samples collected from central and peripheral regions, respectively) compared to the values obtained in group II.

In the isolated primary trophoblast cultures the differences were even more evident $(p<0.02)$ and the mean change in OXTR expression in group I comprised approximately 6.9-fold increase and 6.5-fold increase (the samples collected from central and peripheral regions, respectively) compared to the values obtained in group II.

(Continued on next page)
\end{abstract}

\footnotetext{
* Correspondence: Dszukiewicz@hotmail.com

'Department of General \& Experimental Pathology with Centre for Preclinical

Research and Technology (CEPT), Medical University of Warsaw, ul.

Pawinskiego 3C, 02-106 Warsaw, Poland

Full list of author information is available at the end of the article
} 
(Continued from previous page)

Conclusions: Upregulation of OXTR within placental trophoblast cells localized close or adherent to uterine wall may play a crucial role in labor with efficient contractile activity (vaginal delivery). Further studies may disclose if this local OXT/OXTR signaling is utilized in the third stage of labor to elicit placental detachment or contribute in a more versatile way throughout the labor period.

Keywords: Oxytocin, Oxytocin receptor, Trophoblast, Human placenta, Myometrial contractility, Uterine contractions

\section{Background}

Normal onset of labor with maintained regular intrauterine contractions leading to shortening and cervical dilation (first stage), expulsion of the fetus (second stage) and placental delivery followed by uterine contraction and retraction (third stage) are needed for successful pregnancy outcome [1]. Some mechanism that trigger the contractile activity of the myometrium are still not clearly understood, including those responsible for detachment of the placenta [2]. Local interactions between the two adjacent surfaces: placental (the maternal side) and the corresponding decidual/myometrial surface area would be considered extremely significant in terms of ability to maintain pregnancy to term and the proper course of parturition [3].

Interestingly, the role of oxytocin (OXT) seems to be more complex than it was suggested before. This nonapeptide (or more precisely the octapeptide amide with amino acid sequence: Cys-Tyr-Ile-Gln-Asn-Cys-Pro-LeuGly- $\mathrm{NH}_{2}$ ) was discovered initially as a neuropeptide $[4,5]$. In the hypothalamus, OXT is synthesized as an inactive precursor protein that is progressively activated by gradual hydrolysis into smaller fragments while it is being relocated along the axon towards the posterior pituitary. The last step of this activation is catalyzed by peptidylglycine alpha-amidating monooxygenase (PAM, EC 1.14.17.3) [6].

Finally, OXT is released to the blood stream and acts directly via axon terminals on OXT receptors (OXTR) in the CNS (e.g. the nucleus accumbens) [7]. Amongst other peptides that act locally (e.g. corticotropin-releasing hormone, and dynorphin), OXT and vasopressin are the only known hormones secreted by the human neurohypophysis to act at a distance [8].

The structure of OXT is higly conserved in placental mammals. However, in some new world primates, a novel structures of OXT have been reported [9]. OXT, and another very similar (differs by only two amino acids) nonapeptide - vasopresin, were isolated and synthetized in 1953 by Vincent du Vigneaud [10, 11]. OXT has a disulfide bond between the two cysteines, and reduction of the disulfide bond inactivates OXT [5].

An intricate interplay between OXT and OXTR is responsible for various functions of OXT acting as neuroregulator or in a paracrine manner in ovary or uterus, including materno-placento-fetal unit $[1,6]$. Despite of the fact that OXT evokes uterine contractions, some discrepancies about the exact role of OXT in parturition have arisen in the mid 1980s, after the first suggestion that OXT synthesis also occurs in peripheral tissues $[12,13]$. Outside the brain, synthesis of mRNA encoding OXT was demonstrated in many human tissues, including intrauterine tissues in advanced gestation, principally in the decidua but also in the placental membranes (chorion, amnion) [14, 15]. These findings may lead to suggestion that paracrine or autocrine role rather than, or in addition to, an endocrine role of OXT should be expected in respect of contractile activity of the human uterus [16, 17]. So if OXT is not considered solely as an endorine factor, many previous conclusions about its effect on the course of subsequent stages of pregnancy and labor should be retested [6].

In contrast to vasopresin, OXT is known to have only one type of specific, high-affinity receptor (OXTR). The human OXTR mRNAs were found to be of two sizes, $3.6 \mathrm{~kb}$ in breast and $4.4 \mathrm{~kb}$ in ovary, endometrium, and myometrium. The OXTR gene is present in single copy in the human genome and was mapped to the gene locus 3p25-3p26.2 [18, 19] OXTR belongs to the rhodopsin-type (class I) G protein (Goq11)-coupled receptor family that requires $\mathrm{Mg}^{2+}$ and cholesterol [20]. OXTR is coupled to phospholipase C (PLC, EC 3.1.4.11) which controls the generation of diacylglycerol (DAG) and inositol 1,4,5 - trisphosphate (InsP3). Thus, after activation of OXTR, inositol triphosphate triggers an increase in $\mathrm{Ca}^{2+}$ influx from both extracellular and intracellular stores, whereas DAG stimulates activation of protein kinases type C (PKC, EC 2.7.11.13), which phosphorylates unidentified target proteins. The OXTR has a weak ligand selectivity profile. It was proved that OXTR is able to couple to different $G$ proteins, including $G_{i}$ and $\mathrm{G}_{\mathrm{s}}$ proteins [21].

Expression of OXTR in the pregnant and non-pregnant myometrium differs significantly $[22,23]$. Activation of functional OXTRs stimulates cell proliferation in human placental trophoblast and choriocarcinoma cell lines [24]. Increased density of OXTR and/or augmented sensitivity to OXT were postulated as prerequisites of effective parturition with normal induction of uterine contractions $[22,25]$. These changes observed at transcription and 
protein levels during pregnancy resulting in heterogenous OXTR expression in the term myometrium [22].

However, there are still serious controversies about exact role of OXTR in the initiation of uterine contractions in term pregnancy. It is not known precisely how the increase in OXTR mRNA concentrations in the myometrium and decidua is regulated and what is the contribution of OXTR within the placental tissue. Both, existence of a desensitization mechanism for post-transcriptional regulation of the OXTR, and interplay between OXT and prostaglandins $\left(\mathrm{PGF}_{2 \alpha}, \mathrm{PGE}\right)$ may be rather responsible for the crucial stage of the liberation of uterine contractions at term [26, 27]. Quite recently the new approach has arisen suggesting that OXT and OXTR interactions are crucial to preserve myocyte regeneration and proliferation rather than induction of the uterine contractile activity [28].

Expression of OXTR in the placental term trophoblast cells of the maternal side, contiguous to the uterine wall has not yet been analyzed thoroughly in humans in the context of contractile activity of the uterus. Development of a suitable model for investigation of OXTR expression in the cytotrophoblast enabling a novel approach to this issue may yield interesting contribution to our understanding of the third stage of labor, above all else may shed some light on the mechanism of placental detachment. The interactions between placental prostaglandins and OXT, for example, have been discussed comprehensively, however, not in relation to trophoblast pool of OXTR [29].

The aim of this study was to examine comparatively OXT contents in the placental tissue adjacent to the uterine wall and expressions of OXTR in this tissue and corresponding isolated placental trophoblast cells obtained after normal labors at term and after cesarean sections performed without spontaneously induced uterine contractions (group I and II, respectively).

\section{Methods}

The study was conducted in compliance with international and local laws of human experimentation and was officially approved by local ethics committee of the Medical University of Warsaw (ethical clearance approval number: KB21/2011). Each woman has signed a written consent for the use of her placentae as the source of trophoblast cells. Strictly speaking, this work has been carried out in accordance with The Code of Ethics of the World Medical Association (Declaration of Helsinki) for experiments involving humans, and the Uniform Requirements for manuscripts submitted to Biomedical Journals have been fulfilled.

\section{Placental tissue collection}

Fourteen placentae obtained from nulliparas after natural unifetal vaginal births (group I) were compared with equal number of gestationally matched placentae collected after elective cesarean sections (group II). The indications for cesarean section were high-grade myopia in pregnant woman and breech presentation of the fetus. More detailed clinical characteristics of the two homogenous (except for the mode of delivery) groups are given in Table 1 . As previously mentioned, uterine contractile activity was not observed in group II.

From each collected placenta, three specimens were excised in a standardized manner from the region contiguous to the maternal surface of the placenta: the first one - from the central part, the next two - from peripheral regions of the placental maternal surface (Fig. 1). All placentae used in this study were complete and characterized by a central umbilical cord insertion. In all cases enrolled in group I, expulsion of the placenta was preceded by its normal separation (so-called shiny Schultz) [30]. The management of the third stage of labor was active with umbilical cord clamped immediately after delivery of the newborn and oxytocin (5 IU) administered i.v. to the mother. Similarly, oxytocin (5 IU) was administered during cesarean section following delivery of the newborn. The tissue material obtained by this procedure was subjected either to freezing in carbon dioxide snow for OXT concentration measurement or served as a source of cytotrophoblast cells for the development of in vitro cultures. Appropriate specimens were also embedded in paraffin and used for for immunohistochemistry of OXTR.

\section{Measurement of OXT content}

Concentration of OXT was estimated in the frozen placental excisions. During this procedure, the tissue was kept on ice. Before obtaining a lysate, the material was initially cut into about $1 \mathrm{~mm}^{3}$ cubes by using a razor blade on a glass plate held on ice. To perform a gentle cell disruption, the cubes were then transferred into a hand-held Potter S homogenizer from Sartorius ${ }^{\text {Tx }}$ Stedim Biotech GmbH. The liquefied tissue was poured into the $1.5 \mathrm{~mL}$ tubes and centrifuged at $10,000 \mathrm{xg}$ for $3 \mathrm{~min}$ at $4{ }^{\circ} \mathrm{C}$. The clear supernatant was subjected to in vitro quantitative measurement of OXT in the placental tissue homogenates by an enzyme immunoassay with colorimetric detection. The Oxytocin ELISA-Kit - (Cat. no. ADI-901-153A-0001) - Enzo Life Sciences, Inc. (USA) was applied with declared by manufacturer sensitivity level at $15 \mathrm{pg} / \mathrm{ml}$ (detection range: $15.6-1.000 \mathrm{pg} / \mathrm{ml}$ ). This species independent kit uses a polyclonal antibody to OXT to bind, in a competitive manner, the OXT in the standard or sample or an alkaline phosphatase molecule which has OXT covalently attached to it. Following simultaneous incubation at $4{ }^{\circ} \mathrm{C}$, the excess reagents are washed away and subtrate is added. After a short incubation time the enzyme reaction is stopped and the yellow color generated read on a microplate reader (Biochrom Asys UVM-340 
Table 1 Clinical characteristics of the two groups studied (median and range). The groups may be treated as homogenous, except for the mode of delivery

\begin{tabular}{|c|c|c|}
\hline Parameter & $\begin{array}{l}\text { Group I } \\
\text { (vaginal delivery) }\end{array}$ & $\begin{array}{l}\text { Group II } \\
\text { (cesarean section) }\end{array}$ \\
\hline Number of patients/newborns/placentas/specimens obtained $(N)$ & $14 / 14 / 14 / 42$ & $14 / 14 / 14 / 42$ \\
\hline Parity & 0 & 0 \\
\hline Gestational age in days (median; range) & $283 ; 269-290$ & $276 ; 265-282$ \\
\hline Preterm uterine contractions & Not reported & Not reported \\
\hline Contractile activity of the uterus at time of delivery & Present and effective & Not present \\
\hline Oxytocin treatment in the first and second stage of labour & Not used & Not used \\
\hline Blood pressure during pregnancy & All records within normal range ${ }^{a}$ & All records within normal range $e^{a}$ \\
\hline Proteinuria during pregnancy & Not present & Not present \\
\hline Liver blood tests (aminotransferases enzymes, AST and ALT levels) & Within normal range ${ }^{b}$ & Within normal range ${ }^{b}$ \\
\hline Diabetes during pregnancy & Not present & Not present \\
\hline Body mass index $<21$ or $>35$ & None & None \\
\hline $\begin{array}{l}\text { Mother's blood (III trimester): hematocrit ( } \mathrm{Ht} \text { ), } \\
\text { hemoglobin ( } \mathrm{Hb} \text { ), red blood cell (RBC) count, } \\
\text { mean cell hemoglobin concentration (MCHC) }\end{array}$ & All within normal ranges ${ }^{c}$ & All within normal ranges ${ }^{c}$ \\
\hline Other identified risk factors & None & None \\
\hline Birth weight in grams (median; range) & 3380; 2990-3730 & $3190 ; 2850-3860$ \\
\hline 5-minute Apgar score below 8 points ${ }^{d}$ & None & None \\
\hline Sex of newborns (M, male; F, female) & $7 M+7 F$ & $8 M+6 F$ \\
\hline Weight of placenta in grams (median; range) & $660 ; 559-790$ & $650 ; 550-810$ \\
\hline
\end{tabular}

${ }^{\mathrm{a}}$ The normal range of blood pressure was defined as systolic pressure between 100 and $140 \mathrm{mmHg}$, and diastolic pressure between 60 and $90 \mathrm{mmHg}$

${ }^{\mathrm{b}}$ The normal range of values for AST is 5-40 units per liter of serum and the normal range of values for ALT is 7-56 units per liter of serum

${ }^{\mathrm{C}} \mathrm{Hb}$ levels 10.0-13.5 g/dl, RBC count 3.2-4.4 million/ $\mu \mathrm{l}, \mathrm{MCHC} 319-355 \mathrm{~g} / \mathrm{L}, \mathrm{Ht}$ 31-41 \%

${ }^{\mathrm{d}}$ Apgar score is determined by evaluation newborn on five criteria that form a backronym (Appearance, Pulse, Grimace, Activity, Respiration) on scale from "0" to " 2 ". The scores are added up and the total sum is an Apgar score. Scores 7 and above are generally nomal, 4-6 fairly low, and 3 and below generally regarded as critically low

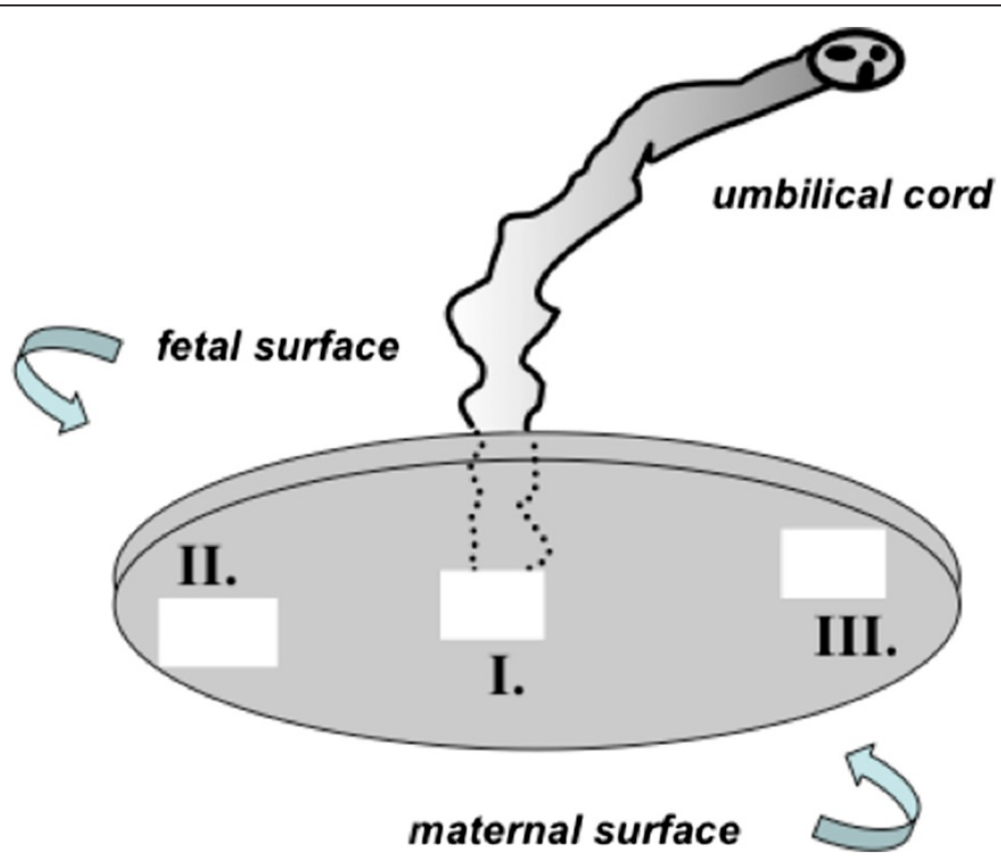

Fig. 1 Location of samples (I - III) collected from the maternal surface of the placenta. The mean weight of the sample: $49.76 \pm 4.54 \mathrm{~g}$ 
Microplate Reader by Biochrom Ltd., Cambridge, UK) at $405 \mathrm{~nm}$. The intensity of the bound yellow color was inversely proportional to the concentration of OXT in either standards or samples. Thus, the measured optical density was used to calculate the concentration of OXT. Relatively low (not exceeding 7-7.5 \%) cross reactivity with $\mathrm{Arg}^{8}$-vasotocin (vasopressin) and very low reactivity for other related molecules $(<0.02 \%)$ provided confidence in OXT assay results. Finally, the concentration of OXT measured by ELISA were converted into ng/g of wet placental tissue. The intra-assay precision (\% CV) amounted to 11.4 and the inter-assay variance (\% CV) was 16.1 .

\section{Trophoblast in vitro cultures}

Concurrently with the measurement of OXT content, preparation of the cell cultures has begun. Cytotrophoblast cells were isolated using modified Kliman's method as described elsewhere [31-33]. This method is based on trypsin, DNase, and a 5-70 \% Percoll gradient centrifugation. Briefly, immediately after obtaining each specimen of the placental tissue was denuded from decidua fragments and abundantly rinsed with $0.9 \%$ saline to remove all blood. A minced villous tissue was subjected to 30 min enzymatic digestion at $37{ }^{\circ} \mathrm{C}$ during incubation in shaking water bath in CMF-HBSS (calcium- and magnesium-free Hank's balanced salt solution; $10 \times$ stock solution from Life Technologies Inc. Gibco/Brl, NY, USA) with $0.125 \%$ trypsin (Sigma-Aldrich Inc., USA; T8003) and $0.2 \mathrm{mg} / \mathrm{ml}$ deoxyribonuclease I (DNase I Type IV; Sigma; D5025). Such digestion was performed sequentially four times. Each time a freshly prepared digestive mixture (solution) was used to the next placental samples. The cells that were released at every $30 \mathrm{~min}$ step were pooled and filtered through two layers of gauze, and trypsin was inactivated with fetal calf serum (FCS). The filtrates were centrifuged, and the cell pellets were resuspended in PBS.

The resultant cell suspension in supernatant was filtered and fractionated on a 5-70 \% Percoll (Sigma; P1644) gradient centrifugation $(1200 \times \mathrm{g}$ for $25 \mathrm{~min})$. The cell layers incorporated into a range 40-50\% (density $1.048-1.062 \mathrm{~g} / \mathrm{ml}$ ) have been collected, washed and resuspended $\left(1 \times 10^{6}\right.$ cells $\left./ \mathrm{ml}\right)$ in Ham's F12/Dulbeco's modified Eagle's medium (1:1; Sigma; $51445 \mathrm{C}$ ) with $15 \%$ fetal bovine serum (Gibco/Brl; 26140-079), $1 \mathrm{mmol} / \mathrm{l}$ sodium pyruvate, $2 \mathrm{mmol} / \mathrm{l} \mathrm{L}$ glutamine, and $50 \mu \mathrm{g} / \mathrm{ml}$ gentamycin. The cultures were then incubated for 5 days under normoxic $\left(20 \% \mathrm{O}_{2}\right)$ conditions at $37{ }^{\circ} \mathrm{C}$ with $5 \%$ of $\mathrm{CO}_{2}$, with the media changed daily. The purity of the cytotrophoblast culture developed from each placenta was evaluated by immunoperoxidase staining with antibodies against vimentin. Described procedure yielded preparation of a highly purified cytotrophoblast (approx. $95 \%$ pure) with the mean viability $\geq 90 \%$.

\section{Immunohistochemistry of OXTR \\ Placental tissue samples}

Human placenta paraffin $5 \mu \mathrm{m}$ sections were subjected to the immunohistochemical staining. Goat anti-human polyclonal IgG (N-terminal) antibody to OXTR (Abnova Corp. Taipei, Taiwan; ABIN1049194; concentration $9 \mu \mathrm{g} / \mathrm{ml}$ ) was applied as primary and chicken anti-goat IgG (heavy and light chains) as biotinylated secondary antibody (Abnova; PAB10566; $0.5 \% \mathrm{v} / \mathrm{v}$ ). In order to highlight the specifically bound primary antibodies to OXTR, the StreptAB-Complex/HRP Duet (Dako Cytomation, Glostrup, Denmark; K0492) was used, following the protocol recommended by the manufacturer, with 3,3'diaminobenzidine that served as a chromogen. The appropriate negative controls for all immunostainings were established simultaneously by replacement of the polyclonal primary antibody by normal goat preimmune IgG diluted with phosphate buffered saline (PBS), containing $3 \%$ bovine serum albumin at the same protein concentration as that used for the primary antibody.

In view of the fact that OXTR are expressed in vascular endothelial cells, it was presumed that the accuracy of OXTR expression measurement may be significantly influenced by the local differences in density of placental microvessels. To avoid this discrepancy in the results, identification of the vasculature elements in placental sections was performed using endothelial cell maker, rabbit polyclonal antibody anti-CD31 (dilution 1 : 50, Abcam Inc., Cambridge, MA, USA; ab28364). Next, a biotinylated goat anti-rabbit antibody was used as the secondary (Abcam).

Using light microscopy with computed morphometry for quantitative analysis (Quantimet 500C+ image analysis workstation provided by Leica, UK), the vascular/extravascular tissular index (V/EVTI) was estimated in calibrated areas of the placental sections, as described in detail elsewhere [34-36]. Briefly, each specimen (paraffin section) underwent three area analyses repeated by two experienced, independent observers. The single area measured with the picture analyzer amounted to $685254 \mu^{2}$ and the total number of specimens 42 per group. The picture analysis procedure consisted in a measurement of the total vascular area. Consequently, the total lumen area of all types of identified vessels was summed up in both groups. With the purpose of a minimizing disruption caused by technical errors, especially unaxial section of the vessel, the lowest value of Ferret's diameter was accepted as the diameter of single lumen. Thus, V/EVTI represents the ratio, which reflects intensity of vascularization and is most closely correlated with the mean density of placental microvessels. 


\section{Cytotrophoblast}

After 5 days the cultures were terminated, formalin-fixed and paraffin-embedded. The paraffin blocks containing cytotrophoblast cells were cut in $4 \mu \mathrm{m}$-thick sections on a microtome with a disposable blade. Visualization of OXTR was performed using standard immunohistochemical pocedures as already described above (see Chapter 2.4.1.) with exception for V/EVTI.

\section{Expression of OXTR}

In order to assess OXTR expression, a quantitative immunohistochemistry based on morphometric software (Quantimet $500 \mathrm{C}+$ ) was performed twice by two independent researchers. Next, the average values were uploaded into the result recording tab. Intensity of immunostaining was evaluated using mean color saturation parameter and thresholdings in grey-level histograms. Thus, expression of OXTR corresponded to the total immunostained area of examined sections, where the color saturation comprises segmentation/separation criteria for objects. A single analysed image area was $131574 \mu \mathrm{m}^{2}$ (magnification $\times 200$ ). In total, 126 visual fields have been analysed (9 visual fields per placenta) in each studied group.

During comparative measurements of OXTR expression in placental tissue samples in both groups, the vascular density-matched samples were analyzed. In any case, the difference between the mean V/EVTI values not exceeded $\pm 5 \%$ [37]. Morphometric results comprising $90 \%$ confidence intervals were reported as the mean fold changes. Naturally, expression of OXTR in the isolated cells of placental cytotrophoblast was estimated without taking into consideration the vascular density.

Application of highly specific antibodies for immunostaining of OXTR together with quantitative imunohistochemistry made it possible to disclose the nature of interrelationships between contractile activity of the uterus and placental expression of OXTR, including isolated trophoblast cells (Fig. 2).

\section{Statistical analysis}

All statistical analyses were performed using Statistica 8.0 software (Stat-Soft, Poland). Mann-Whitney's U test was applied. The results are expressed as the mean \pm SEM, medians or mean percentage values \pm SEM. The differences between groups I and II (natural vaginal birth vs. cesarean section without contractile activity of the uterus) were deemed statistically significant if $p<0.05$.

\section{Results}

To compare OXT levels in the placenta from vaginal ans CS births, placental extracts were analyzed by a standard

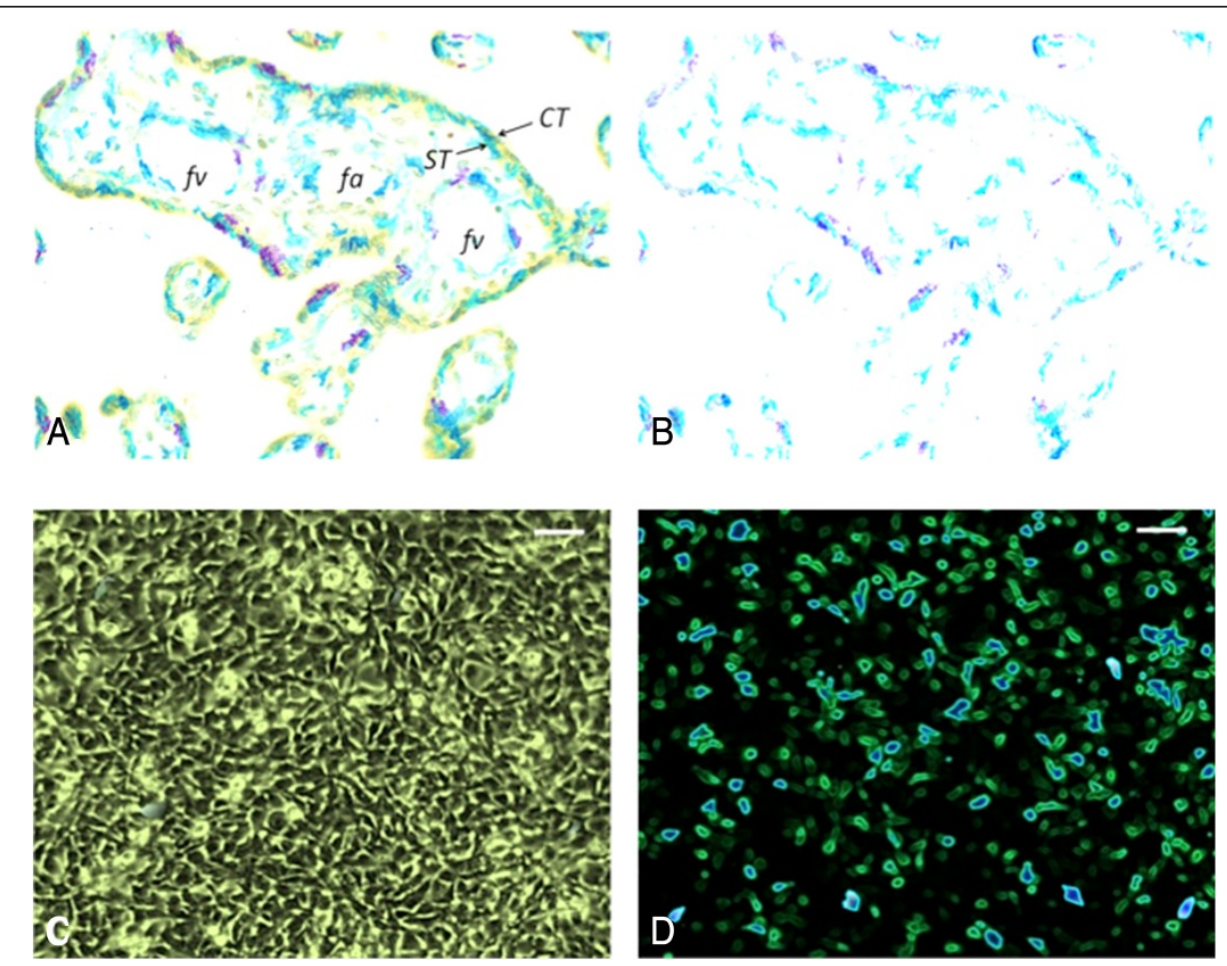

Fig. 2 Immunohistochemical visualization of OXTR. a, b - placental samples (the image captured through optical microscope was subjected to series of digital transformations for morphometric purposes; initial magnification 400x); c - trophoblast cells in culture (phase -contrast image); $\mathbf{d}$ - trophoblast culture immunostained for OXTR (the image digitally transformed for morphometric purposes). The OXTR is represented by the category of blue hues ranged from dark blue to violet blue. Scale bar $=100 \mu \mathrm{m}$. CT - cytotrophoblast, ST - syncytiotrophoblast, fv - fetal vein, fa - fetal artery 
ELISA assay. The results pertaining to OXT content in the placental tissue homogenates are summarized in Fig. 3. There were not significant differences between the groups in respect to the mean OXT concentration in the placental samples obtained from the maternal surface of the placenta. The mean value for the total number of samples in group I amounted to $56.82 \pm 4.78 \mathrm{ng} / \mathrm{g}$ of wet tissue \pm SEM, while in group II the mean OXT content was estimated to be $54.35 \pm 4.93 \mathrm{ng} / \mathrm{g}$ of wet tissue \pm SEM.

It is worth noting that the mean OXT concentration revealed correlation with the location of samples. However, considering that the data are not normally distributed within the groups, the median values (the middle values) have been juxtaposed.

As this may be quite important, in both groups the median value of OXT concentration was significantly $(p<0.05)$ higher in the tissue obtained from the peripheral regions of the maternal surface (Fig. 3b), compared to the samples from the central region (Fig. 3a). In group I (vaginal delivery) the respective median values amounted to $59.97 \mathrm{ng} / \mathrm{g}$ of wet tissue and 47.39 , respectively, while in group II (elective cesarean section) the median values of OXT concentration were estimated to be 59.91, and 43.45 (peripheral and central region, respectively).

Relative changes in the mean OXTR expression in the placental samples and in the trophoblast cultures are shown in Fig. 4 (4a and 4b, respectively). It is clearly visible that in placental samples obtained after vaginal delivery (group I) the mean density of OXTR undergoes significant $(p<0.05)$ upregulation. The mean expression of OXTR in group I was increased by approximately 3.2fold and 3.45-fold (the samples collected from central and peripheral regions, respectively) compared to the values obtained in group II (cesarean section).

The results of comparative evaluation of the mean OXTR expression in the isolated primary trophoblast cultures (Fig. 4b), which means that the influence of the placental vasculature was eliminated, revealed generally the same characteristics of dependencies as already observed for placental samples. However, the differences were even more evident $(p<0.02)$. The mean change in OXTR expression in group I (vaginal delivery) comprised approximately 6.9-fold increase and 6.5-fold increase (the samples collected from central and peripheral regions, respectively) compared to the values obtained in group II (cesarean section).

\section{Discussion}

The control of timing of labor is achieved by complex interactions between mother, fetus and the placenta. Since the contact area of the maternal side of the human placenta is relatively large, this organ may influence uterine contractility throughout a pregnancy. Both local (limited to the site of placental attachment) and all-encompassing interrelationships should be better elucidated [38]. Our results indicate that despite of similar OXT concentration in the placental tissue, the OXTR density may determine onset of the myometrial contractions at the time of delivery. Independent authors already reported that the progress of labor is not related to an increase in OXT concentration, uterine contractions are not associated with changes in plasma OXT concentration and hypocontractile labor does not appear to be the result of a deficit of OXT [39]. These authors concluded that their results do not support a role of OXT during spontaneous labor unless uterine activity is

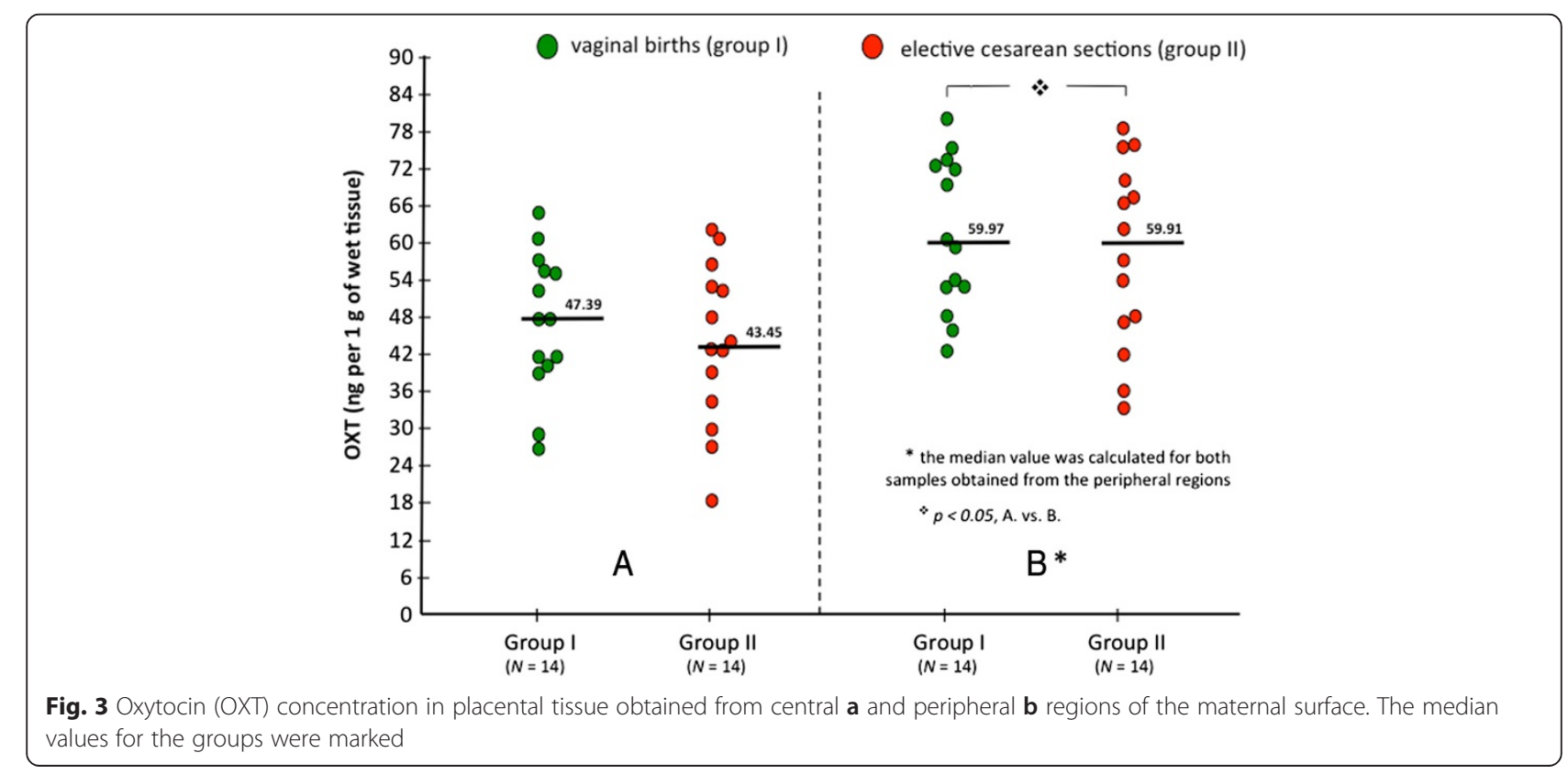




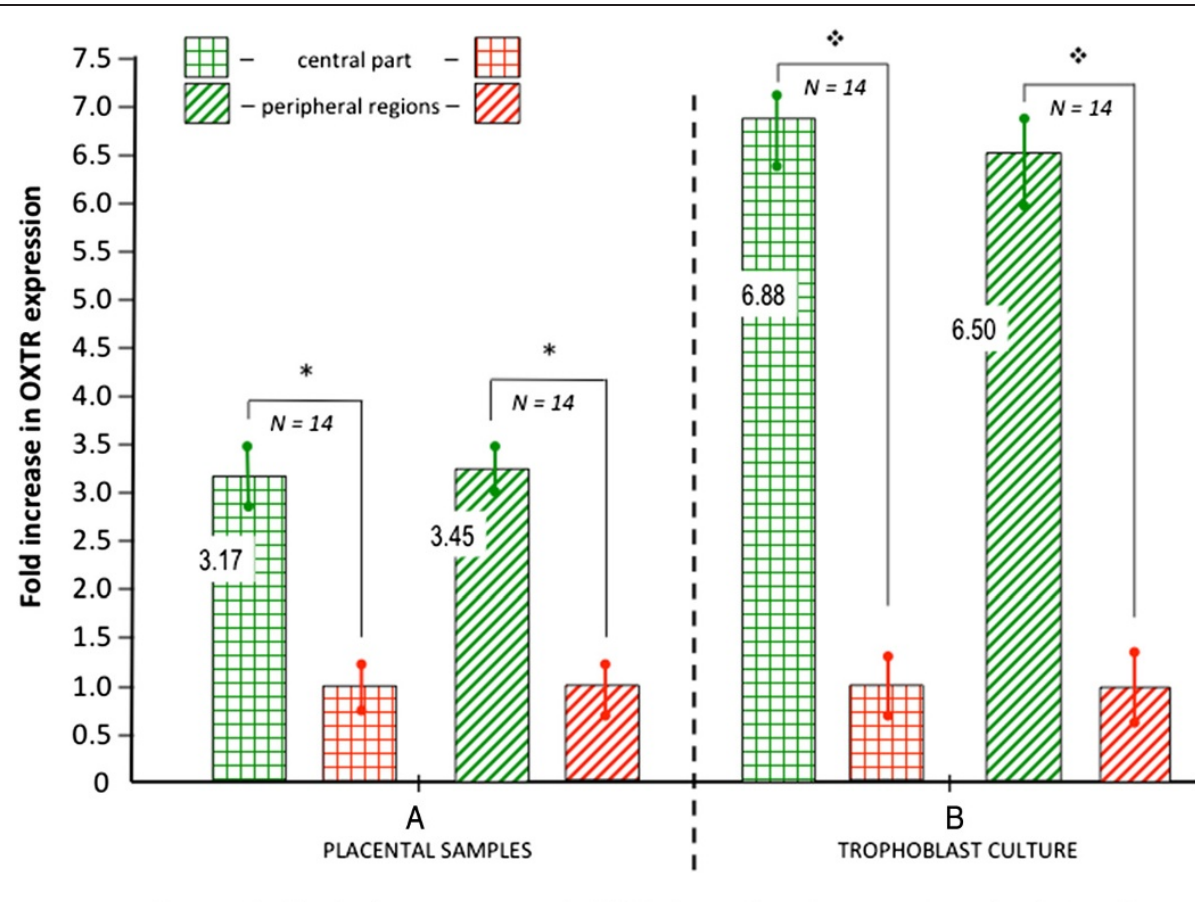

Patterns inside the bars: green - vaginal birth (group I); red - cesarean section (group II

Fig. 4 Group I vs. group II. Mean fold increase in OXTR expression in placental samples $\mathbf{a}$ and the trophoblast cultures $\mathbf{b}$. The data are expressed as mean \pm SEM (* indicates $p<0.05$; indicates $p<0.02$ )

controlled by extremely low plasma hormone concentrations or the uterus becomes sensitive to a constant OXT concentrations [39]. Considering our findings, the latter condition may be caused by region-specific upregulation of OXTR.

The mechanisms utilized to initiate and coordinate contractile activity of the uterus during labor are not known $[1,40]$. Existence of an pacemaker, a site where the action potential (AP) is generated, forms the basis of the canonical AP propagation theory [41]. In all excitable tissues, including uterine smooth muscle cells, AP resulting from the depolarization of the cell membrane (sarcolemma) is the trigger for many intracellular events. First of all, depolarized plasma membrane opens voltage-sensitive ion channels resulting in highly increased entry of extracellular $\mathrm{Ca}^{2+}$ which in turn causes calcium-dependent uterine contractions, especially in calcium-sensitized myometrium [42]. Two types of APs have been observed in myometrial smooth muscle cells: simple AP with depolarization followed by rapid repolarization, and complex AP, where initial depolarization devolves into sustained plateau. It has been suggested, that these different patterns of electrical activity may result from other combinations of ionic currents during the course of given type AP $[43,44]$.

Apart from this, after many years of investigations, the concept of pacemaker is still an open debate since there is no evidence for the presence of cells with such histologic or electrophysiologic characteristics. It was even suggested that all uterine smooth muscle cells have the capacity to act as pacemaker cells, and thereby initiate the contractions [41]. Some researchers have postulated the existence of a unique, subendometrial junctional zone of the myometrium that may generate and regulate these bioelectrical activities [45]. Thus, changed properties of placental cells adjacent to the decidua may significantly affect contractile status of the uterus [46, 47]. As in the uterus, OXT also stimulates production of prostaglandins, including $\mathrm{PGF}_{2 \alpha}$ in the decidua, amnion and cytotrophoblast [48]. It was suggested that estrogen stimulated, local production of OXT within the chorio-decidual and trophoblast cells may contribute to the modulation of AP promoting formation of the complex APs [49].

Calcium sensitization (CS) is considered as a potentially significant functional mechanism for the regulation of uterine contractility. In this condition, especially after stimulation with an agonist such as OXT, a given influx of $\mathrm{Ca}^{2+}$ will cause a larger than expected strenght of contraction [48]. Amongst various uterotonins evaluated for their ability to evoke CS, the effects of OXT have been analyzed thoroughly. It has became evident that CS plays important role in potentialization of the contractile effects of OXT on the human myometrium $[22,49]$. Our results thus suggest that, depending on OXTR expression, the same level of CS produced by similar concentrations of OXT may be sufficient or unable to generate a sustained regular uterine contractions. It should be considered whether 
local functional changes in placental tissue during the short period immediately preceding onset of labor may upregulate OXTR [50]. Because OXT in uterine tissue activates OXTR and another member of the G proteincoupled receptor (GPCR) family, vasopressin $\mathrm{V}_{1 \mathrm{~A}}$ receptor, the local expression of this latter may also influence uterine contractility. However, $\mathrm{V}_{1 \mathrm{~A}}$ receptor affinity and potency of OXT are much lower than those of vasopressin [51].

Immunohistochemical stainings of the placental samples have revealed that OXTR are expressed in both vascular endothelial cells and trophoblast cells (Fig. 3a). In view of this, assessment of OXTR expression performed in placental sections in conjunction with the mean density of placental microvessels (V/EVTI) as well as the use of isolated trophoblast cells in culture are justified in our study. In that way, we were able to demonstrate that perinatal upregulation of OXTR take place predominantly or is even limited to the trophoblast cells located on the maternal side of term placenta. To the best of our knowledge, this is the first publication pertaining to humans aimed at assessing placental OXT concentration in the context of uterine contractility and OXTR density in both placental tissue and isolated trophoblast cells.

Interestingly, in both groups the mean OXT concentration was higher in the peripheral regions of the placenta compared to the central region. It may indicate that significant differences in the basal level of CS could be an important factor influencing contractility of the uterus, possibly during delivery of the placenta. The most commonly observed birth of the placenta by the Schultz Mechanism include the formation of a retroplacental hematoma between the separating placenta and remaining spongiosa layer of the decidua. It facilitates the completion of placental separation [52]. Thus, together with an optimal expression of OXTR, different CS of the myometrium within the placental site evoked by changes in OXT concentration may play a role in the typical mechanism of placental separation during the third stage of labor. It can be speculated that in less common the Matthew Duncan mechanism of placental detachment, as well as in many disorders of the placental stage of labor, expression patterns of OXTR may be different $[2,53]$.

Contrary to above deduction, observed changes in the mean OXT concentration between central and peripheral regions of the maternal surface may be a consequence of dissimilar number of cells in the placental tissue obtained from different locations. This possibility was mentioned by some independent authors $[54,55]$. In addition, to some extent the differences in distribution of placental microvessels and resulted diverse blood supply may contribute to the above results. It should also be taken into account that non-contemporaneous differentiation of the trophoblast in the primary cultures may influence the results pertaining to OXTR expression. The assessment of trophoblast differentiation was not performed in this study.

If we focus on the tissue level, AP propagation theory is sufficient to explain induction of the uterine contractions. However, given the difficulty AP propagation alone has explaining organ-level function, largely forgotten idea of mechanotransduction by pressure-tension sensing should be re-considered $[40,56]$. According to this concept, based on Laplace's Law, the contractions may be controlled by stretch of the uterine wall while significant increase of intrauterine pressure causes increases of wall tension. Next, the optimal level of the wall tension activates contractions throughout the uterus. It is very likely, that both AP propagation from uterine pacemaker and mechanotransduction are complementary and may be helpful with blanket conceptualization of the uterine contraction phenomenon [57]. It is possible that the mechanism of placental detachment from not initially contracting part of the uterine utilizes the pacemaker as the main mechanism of AP activation, while mechanotransduction mechanism is needed for an organ-level coordination of myometrial repetitive, synchronous contractions during normal human labor [58]. Based on our results, we suspect that interaction between OXT and OXTR is an important part of this functional machinery that may be explained using both action potential (AP) and mechanotransduction theories.

In conclusion, the results indicate that upregulation of OXTR within placental trophoblast cells localized close or adherent to uterine wall may play a crucial role in the mechanisms involved in labor with efficient contractile activity. Further studies are needed to disclose if this local OXT/OXTR signaling is utilized in the third stage of labor to elicit placental detachment or contribute in a more versatile way throughout the labor period, including initiation of labor.

\section{Abbreviations \\ AP: Action potential; CMF-HBSS: Calcium- and magnesium-free Hank's balanced salt solution; DAG: Diacylglycerol; CNS: Central nervous system; CS: Calcium sensitization; FCS: Fetal calf serum; GPCR: G protein-coupled receptor; InsP3: Inositol 1,4,5 - triphosphate; OXT: Oxytocin; OXTR: Oxytocin receptor; PAM: Peptidylglycine alpha-amidating monooxygenase; PBS: Phosphate buffered saline; PGE: Prostaglandin E; PGF 2 : Prostaglandin $F_{2 a i}$ PKC: Protein kinases type C; PLC: Phospholipase C; V/EVTI: Vascular/ extravascular tissular index.}

\section{Competing interests}

The authors declare that they have no competing interests.

\section{Authors' contributions}

SD: the study design, cell culture techniques control, quantitative morphometry, statistical analysis, co-supervising all aspects of the project and writing of the manuscript; BA: participation in the study design, data analysis and interpretation, isolation of trophoblast cells; MTK: collection of the informed consents from placental donors; the tissue collection, the initial stages of trophoblast cells isolation procedure; SA: initial stages of trophoblast cell quality assurance and 
monitoring the course of the study, quantitative morphometry; WJ: all data acquisition and assistance in data analysis; SG: collection of the informed consents from placental donors; the tissue collection, the initial stages of trophoblast cells isolation procedure; PM: oxytocin concentration measurement, immunohistochemistry, interpretation of immunostainings; ZJ: co-supervising all aspects of the project, critical review of the manuscript. All authors read and approved the final manuscript.

\section{Acknowledgments}

This research was supported by internal grant 2M2/W1/13 founded by the Medical University of Warsaw. Invaluable advice on the study design provided by Prof. Danuta Maslinska is gratefully acknowledged.

\section{Author details}

'Department of General \& Experimental Pathology with Centre for Preclinical Research and Technology (CEPT), Medical University of Warsaw, ul. Pawinskiego 3C, 02-106 Warsaw, Poland. ²Department of Obstetrics \& Gynecology, Second Faculty of Medicine, Medical University of Warsaw, ul. Kondratowicza 8, 03-242 Warsaw, Poland. ${ }^{3}$ Department of Pathology, Professor Witold Orlowski Public Clinical Hospital, Medical Center for Postgraduate Education, Czerniakowska 231, 00-416 Warsaw, Poland. ${ }^{4}$ Gynecology Clinical Care Unit, Department of Obstetrics and Gynecologic Oncology in Bytom, Medical University of Silesia, ul. Batorego 15, 41-902 Bytom, Poland.

\section{Received: 22 April 2015 Accepted: 11 September 2015 Published online: 16 September 2015}

\section{References}

1. Aguilar HN, Mitchell BF. Physiological pathways and molecular mechanisms regulating uterine contractility. Hum Reprod Update. 2010;16:725-44. doi:10.1093/humupd/dmq016.

2. Wicherek L, Klimek M, Dutsch-Wicherek M, Kolodziejski L, Skotniczny K. The molecular changes during placental detachment. Eur J Obstet Gynecol Reprod Biol. 2006;125:171-5. doi:10.1016/j.ejogrb.2005.05.019.

3. Kota SK, Gayatri K, Jammula S, Kota SK, Krishna SV, Meher LK, et al. Endocrinology of parturition. Indian J Endocrinol Metab. 2013;17:50-9. doi:10.4103/2230-8210.107841.

4. Dale HH. On some physiological actions of ergot. J Physiol. 1906; 34:163-206. doi:10.1113/jphysiol.1906.sp001148

5. Viero C, Shibuya I, Kitamura N, Verkhratsky A, Fujihara H, Katoh A, et al. REVIEW: Oxytocin: Crossing the bridge between basic science and pharmacotherapy. CNS Neurosci Ther. 2010;16:e138-156. doi:10.1111/j. 1755-5949.2010.00185.x.

6. Bealer SL, Armstrong WE, Crowley WR. Oxytocin release in magnocellular nuclei: neurochemical mediators and functional significance during gestation. Am J Physiol Regul Integr Comp Physiol. 2010;299:R452-458. doi:10.1152/ajpregu.00217.2010.

7. Knobloch HS, Grinevich V. Evolution of oxytocin pathways in the brain of vertebrates. Front Behav Neurosci. 2014;8:31. doi:10.3389/fnbeh.2014.00031.

8. Brown CH, Bains JS, Ludwig M, Stern JE. Physiological regulation of magnocellular neurosecretory cell activity: integration of intrinsic, local and afferent mechanisms. J Neuroendocrinol. 2013;25:678-710. doi:10.1111/ jne.12051.

9. Lee $A G$, Cool DR, Grunwald WC, Neal DE, Buckmaster CL, Cheng MY, et al. A novel form of oxytocin in New World monkeys. Biol Lett. 2011;7:584-7. doi:10.1098/rsbl.2011.0107.

10. Taylor Jr SP, Du Vigneaud V, Kunkel HG. Electrophoretic studies of oxytocin and vasopressin. J Biol Chem. 1953;205:45-53.

11. Gordon S, Du Vigneaud V. Preparation of S, S'-dibenzyloxytocin and its reconversion to oxytocin. Proc Soc Exp Biol Med. 1953;84:723-5.

12. Kiss A, Mikkelsen JD. Oxytocin - anatomy and functional assignments: a minireview. Endocr Regul. 2005;39:97-105.

13. Dawood MY, Khan-Dawood FS. Human ovarian oxytocin: its source and relationship to steroid hormones. Am J Obstet Gynecol. 1986;154:756-63. doi:10.1016/0002-9378(86)90450-3.

14. Friebe-Hoffmann U, Baston DM, Hoffmann TK, Chiao JP, Rauk PN. The influence of interleukin-1beta on oxytocin signalling in primary cells of human decidua. Regul Pept. 2007;142:78-85. doi:10.1016/ j.regpep.2007.01.012
15. Blanks AM, Vatish M, Allen MJ, Ladds G, de Wit NC, Slater DM, et al. Paracrine oxytocin and estradiol demonstrate a spatial increase in human intrauterine tissues with labor. J Clin Endocrinol Metab. 2003;88:3392-400. http://dx.doi.org/10.1210/jc.2002-021212.

16. Reis FM, Florio P, Cobellis L, Luisi S, Severi FM, Bocchi C, et al. Human placenta as a source of neuroendocrine factors. Biol Neonate. 2001;79: 150-6. doi:10.1159/000047083.

17. Mitchell BF, Fang X, Wong S. Oxytocin: a paracrine hormone in the regulation of parturition? Rev Reprod. 1998;3:113-22.

18. Michelini S, Urbanek M, Dean M, Goldman D. Polymorphism and genetic mapping of the human oxytocin receptor gene on chromosome 3. Am J Med Genet. 1995;60:183-7. doi:10.1002/ajmg.1320600303.

19. Gimpl G, Reitz J, Brauer S, Trossen C. Oxytocin receptors: ligand binding, signalling and cholesterol dependence. Prog Brain Res. 2008;170:193-204. doi:10.1016/S0079-6123(08)00417-2.

20. Sladek CD, Song Z. Diverse roles of G-protein coupled receptors in the regulation of neurohypophyseal hormone secretion. J Neuroendocrinol. 2012;24:554-65. doi:10.1111/j.1365-2826.2011.02268.x.

21. Koehbach J, Stockner T, Bergmayr C, Muttenthaler M, Gruber CW. Insights into the molecular evolution of oxytocin receptor ligand binding. Biochem Soc Trans. 2013;41:197-204. doi:10.1042/BST20120256.

22. Kimura T, Ogita K, Kumasawa K, Tomimatsu T, Tsutsui T. Molecular analysis of parturition via oxytocin receptor expression. Taiwan J Obstet Gynecol. 2013;52:165-70. doi:10.1016/j.tjog.2013.04.004.

23. Gram A, Boos A, Kowalewski MP. Uterine and placental expression of canine oxytocin receptor during pregnancy and normal and induced parturition. Reprod Domest Anim. 2014;49 Suppl 2:41-9. doi:10.1111/rda.12295.

24. Strunecká A, Hynie S, Klenerová V. Role of oxytocin/oxytocin receptor system in regulation of cell growth and neoplastic processes. Folia Biol (Praha). 2009:55:159-65.

25. Kimura T, Saji F, Nishimori K, Ogita K, Nakamura H, Koyama M, et al. Molecular regulation of the oxytocin receptor in peripheral organs. J Mol Endocrinol. 2003;30:109-15. doi:10.1677/jme.0.0300109.

26. Wathes DC, Borwick SC, Timmons PM, Leung ST, Thornton S. Oxytocin receptor expression in human term and preterm gestational tissues prior to and follwing the onset of labour. J Endocrinol. 1999;161:143-51. doi:10.1677/joe.0.1610143.

27. Kamel RM. The onset of human parturition. Arch Gynecol Obstet. 2010;281:975-82. doi:10.1007/s00404-010-1365-9.

28. Elabd C, Cousin W, Upadhyayula P, Chen RY, Chooljian MS, Li J, et al. Oxytocin is an age-specific circulating hormone that is necessary for muscle maintenance and regeneration. Nat Commun. 2014;5:4082. doi:10.1038/ ncomms5082.

29. Olson DM, Ammann C. Role of the prostaglandins in labour and prostaglandin receptor inhibitors in the prevention of preterm labour. Front Biosci. 2007;12:1329-43. http://ds.doi.org/10.2741/2151.

30. Bair ME, Williams J. Management of the third stage of labor. J Midwifery Womens Health. 2007;4:412-4. doi:10.1016/ j.jmwh.2007.02.019.

31. Kliman HJ, Nestler JE, Sermasi E, Sanger JM, Strauss JF. Purification, characterization, and in vitro differentiation of cytotrophoblasts from human term placentae. Endocrinology. 1986;1 18:1567-82. http://dx.doi.org/10.1210/ endo-118-4-1567.

32. Szukiewicz D, Gujski M, Maslinska D, Szewczyk G, Bachanek M, Maslinski S. Mast cell-derived VEGF and VEGF receptor type 1, 2, and 3 expression in human term trophoblast culture - influence of hypoxia. Inflamm Res. 2005;54 Suppl 1:S82-3. doi:10.1007/s00011-004-0438-2.

33. Szewczyk G, Pyzlak M, Smiertka W, Klimkiewicz J, Szukiewicz D. Does histamine influence differentiation of trophoblast in preeclampsia? Inflamm Res. 2008;57 Suppl 1:S71-2. doi:10.1007/s00011-007-0635-X.

34. Szukiewicz D, Arlena S, Maslinska D, Markowski MW. Placental mast cells (MC) and histamine $(\mathrm{HA})$ in pregnancy complicated by diabetes class $\mathrm{C}$ relation to the development of villous microvessels. Placenta. 1999;20 Suppl 1:503-10. doi:10.1016/S0143-4004(99)80039-3.

35. Huppertz B, Abe E, Murthi P, Nagamatsu T, Szukiewicz D, Salafia C. Placental angiogenesis, maternal and fetal vessels - a workshop report. Placenta. 2007;28(Suppl A):S94-6. doi:10.1016/j.placenta.2007.01.015.

36. Szukiewicz D, Kochanowski J, Pyzlak M, Szewczyk G, Stangret A, Mittal TK. Fractalkine (CX3CL1) and its receptor CX3CR1 may contribute to increased angiogenesis in diabetic placenta. Mediators Inflamm. 2013;2013:437576. doi:10.1155/2013/437576. 
37. Brown KJ, Maynes SF, Bezos A, Maguire DJ, Ford MD, Parish CR. A novel in vitro assay for human angiogenesis. Lab Invest. 1996;75:539-55.

38. Iliodromiti Z, Antonakopoulos N, Sifakis S, Tsikouras P, Daniilidis A, Dafopoulos K, et al. Endocrine, paracrine, and autocrine placental mediators in labor. Hormones (Athens). 2012;4:397-409. doi:10.14310/horm.2002.1371.

39. Thornton S, Davison JM, Baylis PH. Plasma oxytocin during the first and second stages of spontaneous human labour. Acta Endocrinol (Copenh). 1992;126:425-9. doi:10.1530/acta.0.1260425.

40. Young RC, Barendse P. Linking myometrial physiology to intrauterine pressure; How tissue-level contractions create uterine contractions of labor. PLoS Comput Biol. 2014;10:e1003850. doi:10.1371/journal.pcbi.1003850.

41. Lammers WJ. The electrical activities of the uterus during pregnancy. Reprod Sci. 2013;2:182-9. doi:10.1177/1933719112446082.

42. Wray S, Jones K, Kupittayanant S, Li Y, Matthew A, Monir-Bishty E, et al. Calcium signaling and uterine contractility. J Soc Gynecol Investig. 2003:5:252-64. doi:10.1016/S1071-55760300089-3.

43. Khan R, Matharoo-Ball B, Arulkumaran S, Ashford M. Potassium channels in the human myometrium. Exp Physiol. 2001;2:255-64. doi:10.1113/ eph8602181.

44. Bursztyn L, Eytan O, Jaffa AJ, Elad D. Modeling myometrial smooth muscle contraction. Ann N Y Acad Sci. 2007:1101:110-38. doi:10.1196/annals.1389.025.

45. Noe M, Kunz G, Herbertz M, Mall G, Leyendecker G. The cyclic pattern of the immunocytochemical expression of estrogen and progesterone receptors in human myometrial and endometrial layers: characterization of the endometrialsubendometrial unit. Hum Reprod. 1999;1:190-7. doi:10.1093/humrep/14.1.190.

46. Makino S, Zaragoza DB, Mitchell BF, Yonemoto H, Olson DM. Decidual activation: abundance and localization of prostaglandin F2alpha receptor (FP) mRNA and protein and uterine activation proteins in human decidua at preterm birth and term birth. Placenta. 2007;5(6):557-65. http://dx.doi.org/ 10.1016/j.placenta.2006.06.010.

47. Castillo-Castrejon M, Meraz-Cruz N, Gomez-Lopez N, Flores-Pliego A, BeltráMontoya J, Viveros-Alcaráz M, et al. Choriodecidual cells from term human pregnancies show distinctive functional properties related to the induction of labor. Am J Reprod Immunol. 2014;1:86-93. doi:10.1111/aji.12179.

48. Arthur P, Taggart MJ, Mitchell BF. Oxytocin and parturition: a role for increased myometrial calcium and calcium sensitization ? Front Biosci. 2007;12:619-33. http://dx.doi.org/10.2741/2087.

49. Shymgol A, Gullam J, Blanks A, Thornton S. Multiple mechanisms involved in oxytocin-induced modulation of myometrial contractility. Acta Pharmacol Sin. 2006;7:827-32. doi:10.1111/j.1745-7254.2006.00393.x.

50. Blanks AM, Shmygol A, Thornton S. Regulation of oxytocin receptors and oxytocin receptor signaling. Semin Reprod Med. 2007;1:52-9. doi:10.1055/s2006-956775.

51. Koehbach J, O'Brien M, Muttenthaler M, Miazzo M, Akcan M, Elliott AG, et al. Oxytocic plant cyclotides as templates for peptide G protein-coupled receptor ligand design. Proc Natl Acad Sci U S A. 2013;110:21183-8. doi:10.1073/pnas.1311183110

52. Begley CM, Gyte GM, Devane D, McGuire W, Weeks A. Active versus expectant management for women in the third stage of labour. Cochrane Database Syst Rev. 2015;3:CD007412. doi:10.1002/14651858.CD007412.pub4.

53. Rapacz-Leonard A, Raś A, Całka J, Janowski TE: Expression of oxytocin receptors is greatly reduced in the placenta of heavy mares with retained fetal membranes due to secondary uterine atony. Equine Vet J 2015. doi:10.1111/evj.12426

54. Schuhmann RA, Wynn RM. Regional ultrastructural differences in placental villi in cotyledons of a mature human placenta. Placenta. 1980;4:345-53.

55. Castellucci M, Kosanke G, Verdenelli F, Huppertz B, Kaufmann P. Villous sprouting: fundamental mechanisms of human placental development. Hum Reprod Uptade. 2000:5:485-94. doi:10.1093/humupd/6.5.485.

56. Csapo A. The diagnostic significance of the intrauterine pressure. II. Clinical considerations and trials. Obstet Gynecol Surv. 1970;6:515-43.

57. Young RC: Synchronization of regional contractions of human labor; direct effects of region size and tissue excitability. J Biomech 2015, pii: S00219290(15)00068-8. doi:10.1016/j.jbiomech.2015.02.002.

58. Shynlova O, Tsui $P$, Jaffer $S$, Lye SJ: Integration of endocrine and mechanical signals in the regulation of myometrial functions during pregnancy and labour. Eur J Obstet Gynecol Reprod Biol 2009, Suppl 1:2-10. doi:10.1016/ j.ejogrb.2009.02.044.

\section{Submit your next manuscript to BioMed Central and take full advantage of:}

- Convenient online submission

- Thorough peer review

- No space constraints or color figure charges

- Immediate publication on acceptance

- Inclusion in PubMed, CAS, Scopus and Google Scholar

- Research which is freely available for redistribution

Submit your manuscript at www.biomedcentral.com/submit 